A complete electronic version of this article and other services, including high-resolution figures, can be found at:

http://stm.sciencemag.org/content/2/59/59ra87.full.html

Supplementary Material can be found in the online version of this article at: http://stm.sciencemag.org/content/suppl/2010/11/22/2.59.59ra87.DC1.html

This article cites 39 articles, 16 of which can be accessed free:

http://stm.sciencemag.org/content/2/59/59ra87.full.html\#ref-list-1

This article has been cited by 1 articles hosted by HighWire Press; see:

http://stm.sciencemag.org/content/2/59/59ra87.full.html\#related-urls

Information about obtaining reprints of this article or about obtaining permission to reproduce this article in whole or in part can be found at:

http://www.sciencemag.org/about/permissions.dtl 


\title{
Adducin- and Ouabain-Related Gene Variants Predict the Antihypertensive Activity of Rostafuroxin. Part 2: Clinical Studies
}

\author{
Chiara Lanzani, ${ }^{1}{ }^{*}$ Lorena Citterio, ${ }^{1 *}$ Nicola Glorioso, ${ }^{2}$ Paolo Manunta, ${ }^{1}$ Grazia Tripodi, ${ }^{3}$ \\ Erika Salvi, ${ }^{4,5}$ Simona Delli Carpini, ${ }^{1}$ Mara Ferrandi, ${ }^{3}$ Elisabetta Messaggio, ${ }^{1}$ Jan A. Staessen, ${ }^{6,7}$ \\ Daniele Cusi, ${ }^{5,8,9}$ Fabio Macciardi, ${ }^{9,10}$ Giuseppe Argiolas, ${ }^{2}$ Giovanni Valentini, ${ }^{11}$ Patrizia Ferrari, ${ }^{3}$ \\ Giuseppe Bianchi ${ }^{1,3+}$ \\ (Published 24 November 2010; Volume 2 Issue 59 59ra87)
}

\begin{abstract}
Twenty years of genetic studies have not contributed to improvement in the clinical management of primary arterial hypertension. Genetic heterogeneity, epistatic-environmental-biological interactions, and the pathophysiological complexity of hypertension have hampered the clinical application of genetic findings. In the companion article, we furnished data from rodents and human cells demonstrating two hypertension-triggering mechanisms-variants of adducin and elevated concentrations of endogenous ouabain (within a particular range) - and their selective inhibition by the drug rostafuroxin. Here, we have investigated the relationship between variants of genes encoding enzymes for ouabain synthesis [LSS (lanosterol synthase) and HSD3B1 (hydroxy- $\delta$-5-steroid dehydrogenase, $3 \beta$ - and steroid $\delta$-isomerase 1 )], ouabain transport $\{M D R 1 / A B C B 1$ [ATP-binding cassette, sub-family B (MDR/TAP), member 1]\}, and adducin activity [ADD1 (adducin 1) and $A D D 3]$, and the responses to antihypertensive medications. We determined the presence of these variants in newly recruited, never-treated patients. The genetic profile defined by these variants predicted the antihypertensive effect of rostafuroxin (a mean placebo-corrected systolic blood pressure fall of 14 millimeters of mercury) but not that of losartan or hydrochlorothiazide. The magnitude of the rostafuroxin antihypertensive effect was twice that of antihypertensive drugs recently tested in phase 2 clinical trials. One-quarter of patients with primary hypertension display these variants of adducin or concentrations of endogenous ouabain and would be expected to respond to therapy with rostafuroxin. Because the mechanisms that are inhibited by rostafuroxin also underlie hypertensionrelated organ damage, this drug may also reduce the cardiovascular risk in these patients beyond that expected by the reduction in systolic blood pressure alone.
\end{abstract}

\section{INTRODUCTION}

In the companion study (1), we reported data showing that, in both experimental rat and human settings, a polymorphism of $\alpha$-adducin or binding of $\mathrm{Na}$,K-ATPase $\left(\mathrm{Na}^{+}\right.$- and $\mathrm{K}^{+}$-dependent adenosine triphosphatase) by endogenous ouabain increased the activation of Src and phosphorylation of $\mathrm{Na}, \mathrm{K}$-ATPase compared to wild-type $\alpha$-adducin or $\mathrm{Na}, \mathrm{K}-\mathrm{ATPase}$ alone, respectively. Rostafuroxin selectively blunted the excess activation of Src and phosphorylation of Na,K-ATPase induced by either the $\alpha$-adducin variant or the ouabain-bound $\mathrm{Na}, \mathrm{K}$ ATPase and normalized blood pressure (BP) in hypertensive rats. The

\footnotetext{
'Division of Nephrology and Dialysis, Chair of Nephrology, San Raffaele Scientific Institute, Università "Vita-Salute" San Raffaele Hospital, Milan 20132, Italy. ${ }^{2}$ Hypertension and Related Diseases Centre-Association of Urogenital Oncology, University of Sassari, Sassari 07100, Italy. ${ }^{3}$ Prassis sigma-tau Research Institute, Settimo Milanese, Milan 20019, Italy. ${ }^{4} \mathrm{KOS}$ Genetic SRL, Milan 20122, Italy. ${ }^{5}$ Department of Medicine, Surgery and Dentistry, Azienda Ospedaliera San Paolo, University of Milan, Milan 20142, Italy. ${ }^{6}$ Studies Coordinating Centre, Division of Hypertension and Cardiovascular Rehabilitation, Department of Cardiovascular Research, University of Leuven, B-3000 Leuven, Belgium. ${ }^{7}$ Genetic Epidemiology Unit, Department of Epidemiology, Maastricht University, 6200 MD Maastricht, Netherlands. ${ }^{8}$ Graduate School of Nephrology, University of Milan, Milan 20142, Italy. ${ }^{9}$ Genomics and Bioinformatics Unit, Fondazione Filarete, Milan 20139, Italy. ${ }^{10}$ Department of Psychiatry and Human Behavior, University of California, Irvine, CA 92697, USA. ${ }^{11}$ Medical Department Research and Development Division, sigma-tau, Pomezia, Rome 00144, Italy.

*These authors contributed equally to this work.

tTo whom correspondence should be addressed: E-mail: bianchi.giuseppe@hsr.it
}

translation of these experimental findings into the clinical practice depends on two points: (i) the demonstration of functional links between either the mutant $\alpha$-adducin or the ouabain-triggered signaling pathways and gene variants that can be used to classify patients into responders and nonresponders to rostafuroxin, and (ii) whether such gene variants selectively predict the response to rostafuroxin but not to other antihypertensive drugs that act by different mechanisms of action. The activity of the Src-Na,K-ATPase signalosome depends on the functional characteristics of adducin and tissue concentrations of endogenous ouabain (within a narrow window of low concentrations). We therefore "a priori" chose genes coding for adducin isoforms [adducin 1 (ADD1) and $(A D D 3)$ ] or enzymes involved in the ouabain synthesis [lanosterol synthase (LSS) and hydroxy- $\delta$-5-steroid dehydrogenase, $3 \beta$ - and steroid $\delta$-isomerase 1 (HSD3B1)] and transport [adenosine triphosphate (ATP)-binding cassette, subfamily B (MDR/TAP), member 1 (multidrug resistance $1, M D R 1$ )] (hereafter called ouabain genes) as plausible candidates for validation in experimental and clinical settings. As described in detail elsewhere $(2,3)$, mutant adducins and ouabain play a critical role during the transition from normotension to hypertension in both rats and humans. Indeed, patients or rats with long-standing hypertension develop structural changes that may superimpose and mask the peculiar genotype-phenotype relationships characterizing the onset of hypertension $(3,4)$. Moreover, 1 month of drug washout (as usually used in clinical trials on hypertension) is not sufficient to remove the 
effect of the previous therapy $[(5,6)$ and Supplementary Material]. For these reasons, we chose to enroll also a subset of newly discovered and never-treated patients with hypertension of recent onset as the most appropriate cohort for testing the BP-lowering effect of rostafuroxin.

Here, we have translated our experimental data to patients by (i) examining the choice of the gene variants predicting the rostafuroxin response with published [(7-10) and Supplementary Material] and new experimental data by testing whether the LSS gene variant (rs2254524) affects ouabain synthesis, and (ii) carrying out two clinical trials. For the first trial, we performed a double-blind crossover study (study 1) in newly discovered and never-treated patients, or previously treated patients after 1 month of washout, as described in detail elsewhere (11), and compared the effect of rostafuroxin on hypertension in patients with and without the gene variants of interest. In the second trial, we performed a controlled study (study 2), in which newly discovered and never-treated patients received two reference antihypertensive drugs [losartan, an angiotensin II receptor antagonist, and hydrochlorothiazide (HCTZ), a $\mathrm{Na}-\mathrm{Cl}$ cotransporter blocker]. Genetic characteristics of these patients were unknown to both physicians and patients.

A

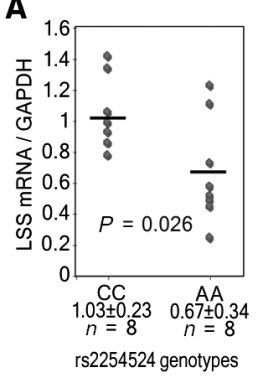

D

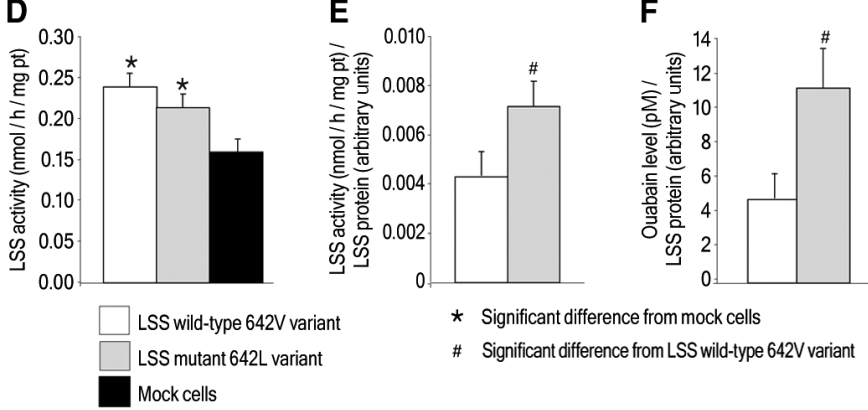

Fig. 1. Expression analysis of LSS polymorphism in human kidneys and H295R-transfected cells. H295R cells were transfected with wild-type (642Val) and mutant (642Leu) variant of LSS rs2254524 or an empty vector (mock). (A) Scatter plot of LSS mRNA expression in renal cortex by QRT-PCR adjusted to GAPDH level (mean $\pm \mathrm{SEM}, n=$ number of samples); horizontal lines, group mean. $P$ value denotes statistically significant difference between the homozygous samples for rs2254524. (B) LSS mRNA levels in transfected cells were obtained by QRT-PCR; data are normalized for GAPDH and expressed as arbitrary units relative to mock level. (C) LSS protein levels in transfected cells were quantified by Western blotting of $5 \mu \mathrm{g}$ of total lysate and expressed as arbitrary units of optical density relative to mock level. (D) LSS activity in transfected cells was determined as reported in Materials and Methods, expressed as micromoles of $\left[{ }^{14} \mathrm{C}\right]$ acetate incorporated in lanosterol per hour per milligram of total cell proteins and corrected for squalene monoepoxide content. (E) Ratio between LSS activity and LSS protein (arbitrary units). (F) Ratio between ouabain secretion and LSS protein (arbitrary units). The data in (B) to (F) are means \pm SEM from at least three separate experiments.

\section{RESULTS}

\section{Links between gene variant controlled activities and the hypertensive mechanisms antagonized by rostafuroxin}

The basis of the candidate gene variant selection is given hereafter and in the Supplementary Material. First, we considered the LSS rs2254524 single-nucleotide polymorphism (SNP), a missense polymorphism (Val642Leu; CC = Val and AA = Leu) located in exon 20 of LSS gene. In human kidney tissues, the LSS messenger RNA (mRNA) expression was lower for the mutant AA $(0.67 \pm 0.12$ arbitrary units, $P<0.028)$ than the wild-type CC variant $(1.03 \pm 0.081)$ (Fig. 1A). Analogously, human adrenocortical H295R cells transfected with the LSS rs2254524 mutant 642Leu variant showed a significant decrease of both LSS mRNA and LSS protein expression compared to cells transfected with the wildtype 642 Val variant $(-42$ and $-35 \%$, respectively, $P<0.05$; Fig. $1, B$ and C). LSS activity was significantly increased in both wild-type $(+50 \%, P<$ $0.05)$ and mutant variant-transfected cells $(+32 \%, P<0.05)$ compared to mock cells (Fig. 1D). Similarly, ouabain production (picomoles per liter per microgram of protein) was higher in both wild-type (254.8 \pm 30 , $P<0.05)$ and mutant variant-transfected cells $(196.5 \pm 9.7, P<0.01)$ than in mock cells $(105.7 \pm 12.3)$. However, LSS activity and ouabain concentrations, normalized for LSS protein expression, were higher in mutant 642Leu than in wild-type 642Val-transfected cells [LSS activity: wildtype $642 \mathrm{Val}, 0.004 \pm 0.001$; mutant $642 \mathrm{Leu}, 0.007 \pm 0.001 \mathrm{nmol} /$ hour per milligram of protein per LSS arbitrary units, $P<0.05$ (Fig. 1E); ouabain levels: wild-type $642 \mathrm{Val}, 4.7 \pm 1.4$; mutant $642 \mathrm{Leu}, 10.9 \pm 2.5 \mathrm{pM}$ per LSS protein arbitrary units, $P<0.05$ (Fig. 1F)]. HSD3B1 is involved in ouabain synthesis, as shown by the fact that its silencing reduces ouabain production in an adrenal medullary-derived cell line (10). A series of tag SNPs, representative of a region of genome with high linkage disequilibrium, is associated with BP variation (12). The six tag SNPs in

Table 1. Selected SNPs for candidate loci. SNP ID is a GenBank ID number [National Center for Biotechnology Information (NCBI)]. Gene map position was taken from human NCBI Genome Build 36.3. Minor allele frequency (MAF) is calculated in previously untreated patients.

\begin{tabular}{|c|c|c|c|c|c|}
\hline$n$ & SNP ID & Chromosome & $\begin{array}{c}\text { Chromosome } \\
\text { position }\end{array}$ & Alleles & MAF \\
\hline 1 & $\begin{array}{l}A D D 1 \text { gene } \\
\text { rs4961 }\end{array}$ & 4 & 2876505 & $\mathrm{G} / \mathrm{T}$ & 0.15 \\
\hline 2 & $\begin{array}{l}A D D 3 \text { gene } \\
\text { rs3731566 }\end{array}$ & 10 & 111876079 & $A / G$ & 0.43 \\
\hline 3 & $\begin{array}{l}\text { MDR1 gene } \\
\text { (ABCB1) } \\
\text { rs1045642 }\end{array}$ & 7 & 86976581 & $\mathrm{~T} / \mathrm{C}$ & 0.48 \\
\hline 4 & $\begin{array}{l}\text { LSS gene } \\
\quad \text { rs2254524 }\end{array}$ & 21 & 46438897 & $\mathrm{G} / \mathrm{A}$ & 0.32 \\
\hline 5 & $\begin{array}{r}\text { HSD3B1 gene } \\
\text { rs10923835 }\end{array}$ & & 119811854 & $\mathrm{~A} / \mathrm{T}$ & 0.10 \\
\hline 6 & rs947130 & & 119818255 & $\mathrm{G} / \mathrm{A}$ & 0.31 \\
\hline 7 & rs2236780 & & 119851986 & $\mathrm{G} / \mathrm{A}$ & 0.32 \\
\hline 8 & rs3765945 & & 119852969 & $\mathrm{~T} / \mathrm{C}$ & 0.38 \\
\hline 9 & rs6203 & & 119858681 & $\mathrm{C} / \mathrm{T}$ & 0.36 \\
\hline 10 & rs10802107 & & 119870455 & $\mathrm{C} / \mathrm{A}$ & 0.26 \\
\hline
\end{tabular}


this region (Table 1), examined alone, were not associated with the systolic BP (SBP) response to rostafuroxin. However, when analyzed in combination with MDR1 rs1045642, LSS rs2254524, or ADD1 rs4961, we found that the HSD3B1 rs10923823 yielded a significant interaction with MDR1 rs1045642 $(P<0.005)$ even after correction for multiple comparisons [corresponding false discovery rate (FDR) $q$ value at $0.10, P<0.006]$. The MDR1 rs1045642 affects both the transmembrane transport of cardiac glycosides and the expression of the P-glycoprotein, which controls digoxin and ouabain plasma concentrations $(12,13)$. In the companion paper, we showed that rostafuroxin antagonizes the effects of mutant human $\alpha$-adducin (Trp460 ADD1 rs4961) on Src-Na,K-ATPase interaction and $\mathrm{Na}, \mathrm{K}$ pump endocytosis without affecting the activity of wild-type adducin (Gly460 ADD1 rs4961). The interaction between ADD1 rs4961 and ADD3 rs3731566 is of particular interest because $\alpha(A D D 1)$ and $\gamma(A D D 3)$ adducins work in renal tubular cells as a dimer (2). Therefore, any interaction between these two genes is likely to have biochemical effects. In particular, an epistatic interaction between these two SNPs on human BP has been demonstrated $(14,15)$. Moreover, the effect of $A D D 1$ is modulated by ouabain plasma levels (16). Consequently, the above-mentioned gene variants (Table 1) must be considered either alone or in appropriate combinations to establish the relationship between the genotypes and SBP response to rostafuroxin.

\section{A relationship between the a priori selected gene variants and the SBP response to rostafuroxin, but not losartan and HCTZ}

In the crossover double-blind study (study 1) [Ouabain and Adducin for Specific Intervention on Sodium in Hypertension (OASIS-HT)], 410 patients with at least one evaluation available for each crossover period were considered for the intention-to-treat statistical analysis. The results showed that rostafuroxin did not reduce SBP over placebo at any dose, and the few side effects that did occur did so with similar frequency in the rostafuroxin and placebo groups. The details of this analysis were published elsewhere (17). The results regarding the genotype-BP relationship, included as one of the secondary endpoints of the trial (11), were obtained in the 346 patients who completed the first period of treatment and had their genotypes properly measured. As discussed in the Supplementary Material, a washout period of 1 month is not enough to remove the effect of previous treatments. This, together with the period effect found in all the groups of patients (17) and the carryover effect $(P=0.0016)$ found in carriers of the appropriate genotypes (see Supplementary Material), led us to consider only the first period of the crossover design and to analyze patients with and without the previous treatment as separate groups. The characteristics of these patients with their basal SBP are shown in Table 2A. Table 3 reports the effects of the genotypes listed in Table 1, alone or in combination, or clustered in a profile [defined as any of four selected pairs of variants (Fig. 2A, c-e)], on the SBP response to rostafuroxin or placebo, recorded only during the first period of treatment in never-treated patients. The epistatic nature of the genotype combination effects on the placebocorrected drop in SBP induced by rostafuroxin is supported by the following: (i) the interactions between mutant $A D D 1$ with either plasma ouabain or mutant $A D D 3$ in determining the BP levels (15), (ii) the ouabain tissue concentrations as determined by its synthesis and transport, and (iii) the statistical analyses reported in Table 3. This analysis showed a deviation of the effect of genotype combinations from the corresponding single genotypes. We dealt with the genetic heterogeneity
Table 2. Clinical characteristics at basal time in patients of study 1 (A.1) and study 2 (B.1), and basal mean values of SBP according to the presence or absence of the profile [defined as any of four selected pairs of variants (Fig. 2A, C-e)] in patients of study 1 (A.2) and study 2 (B.2). BMI, body mass index.

A.1

\begin{tabular}{lrcr}
\hline OASIS-HT & Mean & SEM & SD \\
\hline Age (years) & 48.1 & 0.42 & 7.88 \\
Height $(\mathrm{cm})$ & 171.2 & 0.48 & 9.01 \\
Weight $(\mathrm{kg})$ & 78.8 & 0.62 & 11.48 \\
BMI $\left(\mathrm{kg} / \mathrm{m}^{2}\right)$ & 26.8 & 0.14 & 2.58 \\
Basal SBP (mmHg) & 150.1 & 0.54 & 7.41 \\
Gender (male/female) & & $207 / 139$ & \\
\hline
\end{tabular}

\begin{tabular}{lcccc} 
A.2 & & & & \\
\hline OASIS-HT & Profile & Mean & SD & SEM \\
\hline Basal SBP $(\mathrm{mmHg})$ & No & 150.0 & 7.5 & 0.5 \\
& Yes & 151.0 & 8.1 & 0.9 \\
Total & & 150.2 & 7.6 & 0.4 \\
\hline
\end{tabular}

B.1

\begin{tabular}{lrrrrrrrr}
\hline & \multicolumn{3}{c}{ Losartan } & & \multicolumn{3}{c}{ HCTZ } \\
\cline { 2 - 5 } \cline { 7 - 9 } & Mean & SEM & SD & & Mean & SEM & SD \\
\hline Age (years) & 44.8 & 0.80 & 7.95 & & 45.7 & 0.54 & 8.35 \\
Height $(\mathrm{cm})$ & 170.0 & 0.99 & 9.66 & & 172.2 & 0.50 & 7.63 \\
Weight $(\mathrm{kg})$ & 77.2 & 1.11 & 10.95 & & 77.5 & 0.77 & 11.61 \\
BMI $\left(\mathrm{kg} / \mathrm{m}^{2}\right)$ & 26.7 & 0.27 & 2.72 & & 26.0 & 0.19 & 2.95 \\
Basal SBP (mmHg) & 147.9 & 0.60 & 5.96 & & 148.6 & 0.85 & 13.06 \\
Gender (male/female) & & $75 / 23$ & & & $198 / 40$ & \\
\hline
\end{tabular}

B.2

\begin{tabular}{lccccccccc}
\hline & \multicolumn{4}{c}{ Losartan } & & \multicolumn{3}{c}{ HCTZ } \\
\cline { 2 - 4 } \cline { 8 - 10 } & Profile & Mean & SD & SEM & & Mean & SD & SEM \\
\hline Basal SBP $(\mathrm{mmHg})$ & No & 148.1 & 5.9 & 0.6 & & 149.0 & 12.8 & 1.0 \\
& Yes & 146.8 & 6.7 & 1.9 & & 147.4 & 13.7 & 1.7 \\
Total & & 147.9 & 6.0 & 0.6 & & 148.6 & 13.1 & 0.8 \\
\hline
\end{tabular}

controlling the ouabain tissue concentrations or the interaction between ouabain and adducin genes by grouping all the patients carrying at least one of the combinations listed in Table 2 as carriers of the profile.

SBP fall was not statistically different among any dose of rostafuroxin and placebo in the overall cohort (Fig. 2A, a), irrespective of the previous treatment (Fig. 2A, b). Conversely, the magnitude of the SBP fall after rostafuroxin was affected by the polymorphisms of the selected gene variants, when considered either alone (LSS rs2254524) (Fig. 3, B versus E: rostafuroxin versus placebo) or in combination (Table 3 and Fig. 2A, d). Although we accounted for the genetic background, no significant dose-effect relationship was detected (Fig. 3, C versus B: 
$0.05 \mathrm{mg}$ of rostafuroxin versus all doses). The influence of the LSS rs2254524 variation on the rostafuroxin effect was already present at a dose of $0.05 \mathrm{mg}(\sim 2 \mathrm{nmol} / \mathrm{kg})$ (Fig. 3C). For these reasons, patients receiving the five doses were pooled (Fig. $3 \mathrm{~B}$ ). This finding resembles that already observed in rats, where a wide range of rostafuroxin doses (from 0.25 to $250 \mathrm{nmol} / \mathrm{kg}$ ) produces similar decreases of SBP, with the dose of $0.025 \mathrm{nmol} / \mathrm{kg}$ being inactive $(18,19)$. In a previous trial, an association between LSS rs2254524 genotype and SBP response to rostafuroxin was detected (2003 trial; Fig. 3A and Supplementary Material). In the present trial, LSS rs2254524 was again found to be associated with the rostafuroxin SBP response (Fig. 3B), even after correction for multiple comparisons $(P=0.008$; correspondent FDR $q$ value at $0.10, P=0.01)$. Therefore, the present findings may be considered as a replication of our previous results and are further strengthened by the demonstration that the LSS rs2254524 SNP affects mRNA, protein expression, and ouabain production in cells transfected with the two variants (Fig. 1, B to F). The genetic influence on the rostafuroxin SBP response shown in Figs. 2A (c) and 3D was confirmed in never-treated patients (Fig. 2A, d versus e). In a subset of previously untreated patients, the average SBP fall in carriers of the profile (Fig. 2A, d, 23\% of the total sample) receiving rostafuroxin or placebo was $-16.18 \pm 2.2$ and $-2.1 \pm$ $2.3 \mathrm{mmHg}\left(P=1.8 \times 10^{-8}\right)$, respectively. One-third of never-treated patients had a fall of SBP on rostafuroxin of $11.7 \mathrm{mmHg}$ or more. Assuming this cutoff, the genetic profile is able to predict the inclusion or the exclusion from this tertile with an odds ratio of 9.1 (confidence interval, 3.22 to $25.73 ; P=6.1 \times 10^{-6}$ ).

The controlled study (study 2 ) was carried out in newly discovered and never-treated patients. The patients were assigned to losartan or HCTZ as described in Materials and Methods. The genotypes of pa- tients were unknown to both physicians and patients. The baseline characteristics and SBP of the patient groups are given in Table 2 (B.1 and B.2). The values for the SBP fall after 4 weeks of treatment in carriers of the profile and in noncarriers after losartan and HCTZ were as follows: $-10.6 \pm 3.3$ versus $-13.1 \pm 1.04 \mathrm{mmHg}$ or $-8.5 \pm 1.5$ versus $-7.8 \pm 0.96 \mathrm{mmHg}$, respectively (Fig. $2 \mathrm{~B}$, a and b). For comparison, see rostafuroxin effect in Fig. 2B (c).

\section{DISCUSSION}

Here, we have shown that variants within five genes previously stated (11) that code for proteins involved in the hypertensive molecular mechanisms underlying the interactions of either $\alpha$-adducin or ouabain with Src-Na,K-ATPase predict the SBP response to rostafuroxin, a selective inhibitor of such interactions, but not to losartan and HCTZ. We considered gene variants thought to affect ouabain synthesis (LSS and HSD3B1) and transport (MDR1), rather than ouabain plasma concentrations itself. As previously shown for the renin-angiotensin-aldosterone and other hormonal systems, the plasma concentration of a hormone does not reflect the paracrine and autocrine changes that occur, for example, as a result of different body $\mathrm{Na}^{+}$concentrations (20). These findings also apply to ouabain, which is also produced by peripheral tissues (9). When compared to carriers of the wild-type ADD1Gly460 allele, hypertensive carriers of the mutant $A D D 1$ Trp460 allele (rs4961) have an increased risk of developing (i) cardiovascular events, as shown in 10 independent studies (21-30), and (ii) hypertension and/or intermediate phenotypes leading to cardiovascular or renal damage (2), when the ACE I/D (angiotensin I converting enzyme insertion/deletion) polymorphism is taken

Table 3. Significant interactions among SNPs on SBP fall after rostafuroxin and placebo. ns, not significant.

\begin{tabular}{|c|c|c|c|c|c|c|c|}
\hline Genes & Genetic variant & Patients $(\%)^{*}$ & $\begin{array}{c}\text { SBP fall (mmHg, } \\
\text { mean } \pm \text { SEM) } \\
\text { placebo }\end{array}$ & $\begin{array}{l}\text { SBP fall }(\mathrm{mmHg} \text {, } \\
\text { mean } \pm \text { SEM) } \\
\text { rostafuroxin }\end{array}$ & $\begin{array}{l}\text { Placebo-corrected } \\
\text { rostafuroxin effect } \\
\text { (mmHg_mean) }\end{array}$ & $r^{2+}$ & $P^{\ddagger}$ \\
\hline LSS & rs2254524 AA & 11 & $-7.43 \pm 5.24$ & $-17.23 \pm 2.82$ & -9.80 & 0.152 & ns \\
\hline LSS & rs2254524 CA+AA & 53 & $-7.11 \pm 1.81$ & $-8.82 \pm 1.74$ & -1.71 & 0.005 & ns \\
\hline$A D D 1$ & rs4961 GT+TT & 28 & $-9.30 \pm 2.56$ & $-5.54 \pm 2.34$ & +3.76 & 0.022 & ns \\
\hline$A D D 3$ & rs3731566 GG & 18 & $-6.27 \pm 3.02$ & $-9.79 \pm 2.61$ & -3.52 & 0.023 & ns \\
\hline MDR1 & rs1045642 CC & 23 & $-7.32 \pm 2.70$ & $-9.43 \pm 2.88$ & -2.11 & 0.007 & ns \\
\hline HSD3B1 & rs10923835 AT+Tा & 20 & $-7.12 \pm 2.15$ & $-7.41 \pm 3.05$ & -0.29 & 0.0002 & ns \\
\hline LSS and $A D D 1$ & $\begin{array}{l}\text { LSS rs2254524 AA and } \\
\text { ADD1 rs4961 GT+TT }\end{array}$ & 4.6 & $5.18 \pm 3.41$ & $-24.50 \pm 5.13$ & -29.67 & 0.798 & 0.003 \\
\hline$A D D 1$ and $A D D 3$ & $\begin{array}{c}\text { ADD1 rs4961 GT+T and } \\
\text { ADD3 rs3731566 GG }\end{array}$ & 5.6 & $-3.25 \pm 3.95$ & $-17.46 \pm 4.32$ & -14.21 & 0.396 & 0.038 \\
\hline LSS and MDR1 & $\begin{array}{l}\text { LSS rs2254524 CA+AA and } \\
\text { MDR1 rs1045642 CC }\end{array}$ & 11 & $-3.96 \pm 3.67$ & $-16.91 \pm 3.33$ & -12.95 & 0.220 & 0.032 \\
\hline$M D R 1$ and $H S D 3 B 1$ & $\begin{array}{l}\text { MDR1 rs1045642 } \Pi \text { and } \\
\text { HSD rs10923835 AT+Tा }\end{array}$ & 5.6 & $-0.10 \pm 5.39$ & $-17.40 \pm 3.35$ & -17.30 & 0.482 & 0.018 \\
\hline Profile & Any of the selected pairs of variants ${ }^{+\dagger}$ & 23 & $-2.06 \pm 2.06$ & $-16.18 \pm 2.24$ & -14.12 & 0.329 & $3.77 \times 10^{-5}$ \\
\hline
\end{tabular}

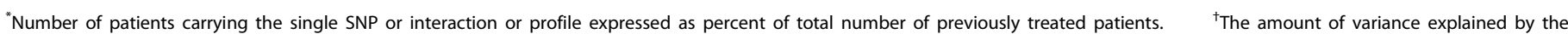

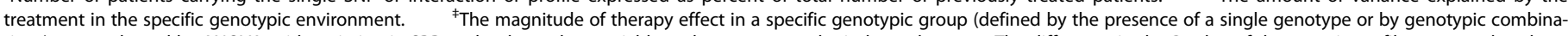

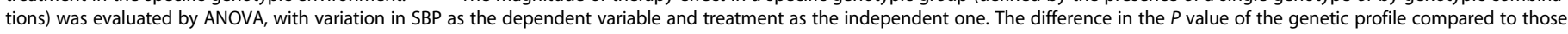

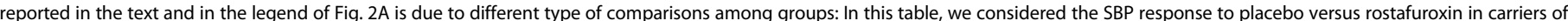

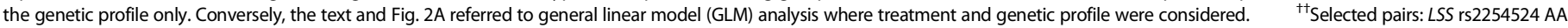
and ADD1 rs4961 GT+TT; ADD1 rs4961 GT+TT and ADD3 rs3731566 GG; LSS rs2254524 CA+AA and MDR1 rs1045642 CC; MDR1 rs1045642 TT and HSD rs10923835 AT+TT. 
into account. Similarly, ouabain plasma concentrations are associated with increased cardiovascular risk (31). Therefore, our present data showing that rostafuroxin can lower SBP in such patients suggest that it is possible to reduce the cardiovascular risk in this group. Accordingly, the wellestablished involvement of the Na,K-ATPase-Src-EGFR (epidermal growth factor receptor)-ERK (extracellular signal-regulated kinase) signaling in organ damage (32) and the selective ability of rostafuroxin to antagonize such signaling activation (1) further support the likelihood that rostafuroxin can help prevent organ complications.

Cardiovascular structural changes associated with long-lasting hypertension may superimpose and mask the characteristic genotype-phenotype relationship causing the initial development of hypertension $(3,4)$. Moreover, 1 month of washout is not enough to remove the influence of the previous therapies $[(5,6)$ and Supplementary Material]. These findings, together with the ones shown in Fig. 2A, support the need to include never-treated patients in pharmacogenomic studies lasting only a few weeks. The disregard of this requirement by most studies on pharmacogenomics in primary hypertensive patients may account for the variable data so far published $(33,34)$.

Three recent large genome-wide association studies of hypertension reported many loci with convincing replications (1), but with a small effect size (1 to $3 \mathrm{mmHg}$ ). Epistasis and genetic heterogeneity are not taken into account in these studies $(35,36)$. By considering the combination of genetic variations hypothesized to underlie the triggering mechanism of hypertension and their selective inhibition with rostafuroxin, we obtained an effect size of $-14 \mathrm{mmHg}$ (resulting from the response to rostafuroxin $<-16.18 \mathrm{mmHg}$ ) corrected by the response to placebo $(-2.06 \mathrm{mmHg})$. This SBP fall is clinically relevant: The pretreatment SBP was $150 \mathrm{mmHg}$, and the rostafuroxin-induced SBP drop, corrected by placebo effect, was twice as large as that obtained with diuretics, angiotensin receptor blockers, or $\beta$-blockers in phase II clinical trials (37).

The present study has the following limitations that will need to be addressed in the future. (i) The data have to be replicated on a larger cohort, even though the results on LSS may be considered a replication of a previous finding. Only with an adequate sample size can we compare all the possible combinations of the genes involved in ouabain metabolism. (ii) The mechanistic links between the

A

B

(a)
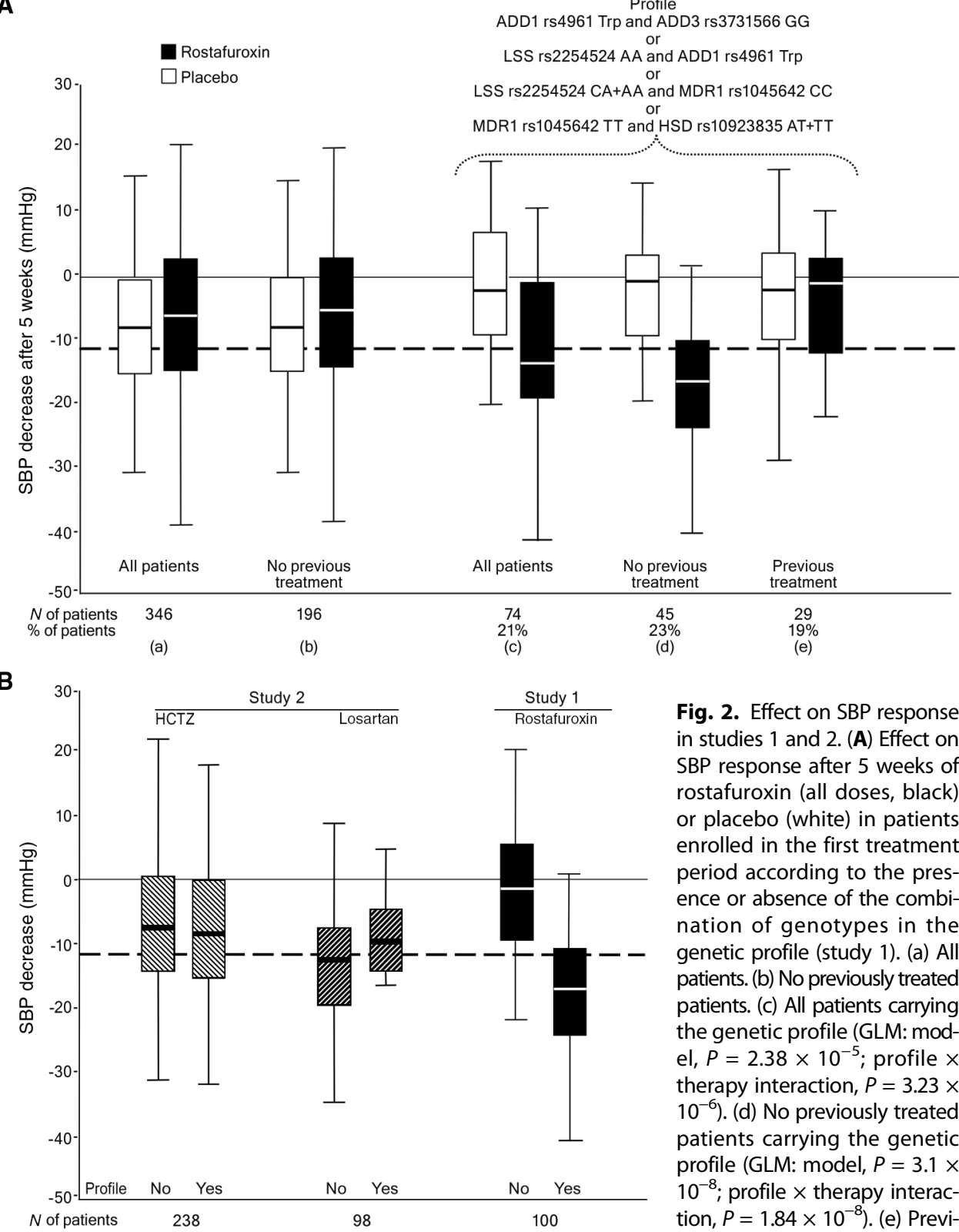

(b)

(c)

Fig. 2. Effect on SBP response in studies 1 and 2. (A) Effect on SBP response after 5 weeks of rostafuroxin (all doses, black) or placebo (white) in patients enrolled in the first treatment period according to the presence or absence of the combination of genotypes in the genetic profile (study 1). (a) All patients. (b) No previously treated patients. (c) All patients carrying the genetic profile (GLM: model, $P=2.38 \times 10^{-5}$; profile $\times$ therapy interaction, $P=3.23 \times$ $10^{-6}$ ). (d) No previously treated patients carrying the genetic profile (GLM: model, $P=3.1 \times$ $10^{-8}$; profile $\times$ therapy interaction, $P=1.84 \times 10^{-8}$ ). (e) Previously treated patients carrying the genetic profile. Percentages

refer to relative group of patients. (B) Effect on SBP response according to the presence or absence of the genetic profile in two studies: 4 weeks of HCTZ (left-oriented black striped) (a) or losartan (right-oriented white striped) (b) (study 2), and 5 weeks of rostafuroxin (all doses, black) (c) (study $1 ; P=7.84 \times 10^{-8}$, one-way ANOVA). The genetic profile consists of the genotypic combinations as indicated. The box plot graph summarizes the statistical measures (median, the 75th and 25th percentiles, and minimum and maximum data values) of the distribution of the SBP decreases $(\mathrm{mmHg}$ ). The continuous line indicates $0 \mathrm{mmHg}$, whereas the thick dotted line represents the first tertile value of SBP decrease after treatment in study 1. 
A

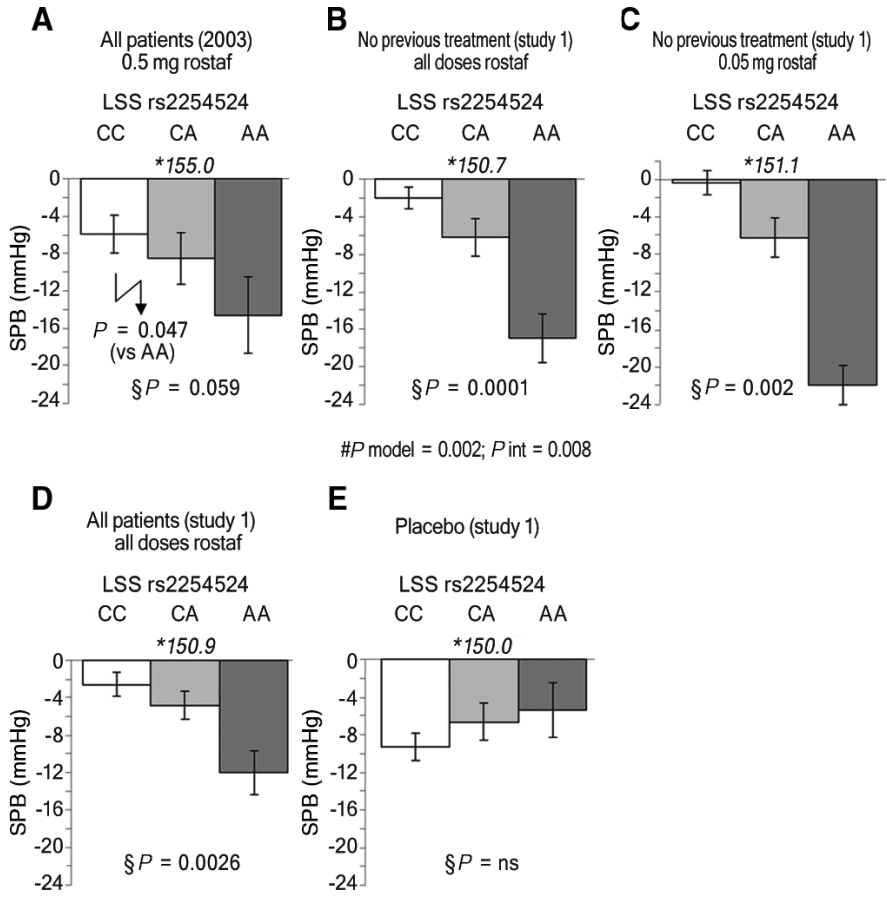

Fig. 3. SBP changes in patients grouped according to the LSS genotypes (rs2254524), rostafuroxin treatment, and placebo. Basal SBP concentrations are indicated by an asterisk. (A) Results from all patients in the 2003 clinical trial (rostafuroxin dose, $0.5 \mathrm{mg} ; n=40$ ). (B) Results from patients with no previous treatment in study 1 (all doses of rostafuroxin; $n=101$ ). (C) Results from patients with no previous treatment in the study 1 (rostafuroxin dose, $0.05 \mathrm{mg}$; $n=27$ ). (D) Results from all patients in study 1 (all doses of rostafuroxin; $n=$ 175). (E) Results from patients with no previous treatment in study 1 (placebo; $n=95$ ). The weak statistical significance in the 2003 trial may be due to the low sample size and the less rigorous exclusion of patients with previous

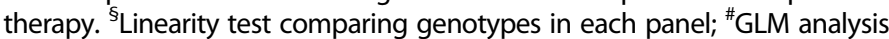
comparing placebo and all doses of rostafuroxin in never-treated patients $[(B)$ versus $(\mathrm{E})] ; P=0.047$, one-way ANOVA, between $C C$ and $A A$ genotypes in $(A)$, rostaf, rostafuroxin.

response to the other antihypertensive drugs may minimize the role of pharmacokinetics.

The goal of drug discovery is to develop molecules that can induce normal function in an abnormal protein variant or molecular mechanism and to translate these molecular effects into a safe and effective therapeutic intervention. The success of this strategy depends on matching the geneticmolecular mechanisms underlying the disease in the target population and those targeted by the drug. With the limitations listed above, the results described here may provide a successful example of this strategy.

\section{MATERIALS AND METHODS}

\section{Clinical trials}

The crossover double-blind study (study 1) (OASIS-HT study) was carried out according to the protocol described by Staessen et al. $(11,38)$. OASIS-HT was conducted according to the rules of good clinical practice of declaration of Helsinki at 39 European centers, which all received approval from the competent Ethics Committees and the Na- tional Regulatory Authorities. OASIS-HT was an early phase 2 dosefinding study. After a run-in period of 4 weeks, eligible patients were randomized to one of five oral doses of rostafuroxin $(0.05,0.15,0.5$, 1.5 , or $5.0 \mathrm{mg} /$ day). Each dose was compared to placebo in a doubleblind crossover experiment with balanced randomization. Treatment was initiated with the active drug and continued with placebo or vice versa. Each double-blind period lasted 5 weeks, with an intermediate visit at 2 weeks and a final visit 3 weeks later. OASIS-HT was therefore a combination of five concurrent crossover studies, one for each dose of rostafuroxin.

In study 2, we investigated the effects of losartan and HCTZ in never-treated patients with and without the genetic profile. Within a large multicenter clinical study aimed at evaluating the association between the genotypes of patients and the response to losartan (4 weeks of treatment) or HCTZ (4 and 8 weeks of treatment), we selected the subset of patients completing the study in Sassari and Milan centers by September 2009. These studies received approval from the competent Ethics Committees of both places. Mild-to-moderate, asymptomatic, never-treated primary hypertensive patients, consecutively visiting the outpatient sections of each participating center, were enrolled if they met all the inclusion and exclusion criteria similar to that of study 1 and after written informed consent for genetic studies was obtained. Except for the crossover design, many aspects of this study were similar to the OASIS-HT study. After a run-in period of 4 weeks, eligible patients were enrolled to receive losartan ( $50 \mathrm{mg} /$ day, orally) or HCTZ ( $12.5 \mathrm{mg} /$ day, orally) for 4 weeks. The quantitative phenotype used for the statistical analyses is the difference in $\mathrm{mmHg}$ between the office SBP at the end of the treatment period (SBP_4) and the office SBP at baseline after 1 month of run-in (SBP_0), which we named DSBP4_0. Briefly, at the screening visit (visit 1 , week -4 ), investigators assessed the eligibility of patients, obtained written informed consent both to participate in the clinical protocol and to undergo a genetic study, performed a complete physical examination, and planned the laboratory/instrumental tests. During the run-in period, the patients remained off treatment. Inclusion and exclusion criteria were to be confirmed at visit 1 (week -4) and visit 3 (enrolment visit, week 0). During the 4-week run-in period, personalized dietary written instructions to ensure a daily intake of 100 to 140 meq of sodium and 50 to 70 meq of potassium were given. This approach was aimed at standardizing the baseline conditions for all patients to minimize the "side effect" arising from life-style differences between patients. Sodium and potassium intake is known to influence intermediate phenotypes such as BP levels and so on. At enrolment (visit 3 , week 0$)$, losartan $(50 \mathrm{mg} /$ day) or HCTZ (12.5 mg/day) was started. The time window allowed for each visit is the scheduled time \pm 3 days. For clinic BP measurement, the appropriate cuff size and the arm with highest $\mathrm{BP}$ were chosen at visit 1 . Certified electronic devices were used. At each visit, office BP was measured in the sitting position after 5-min rest, five times, and with a 30 - to 60 -s interval between readings. Only the last two values were considered for the analysis.

\section{SNP selection and human genotyping}

Four SNPs (rs4961, rs3731566, rs1045642, and rs2254524) were selected for their functional characteristics, explained in the Results section, whereas the remaining six SNPs were derived from the International HapMap Project Web site and tagged HSD3B1 gene (Table 2) (39).

SNP genotyping was performed on genomic DNA extracted from blood samples by means of the $5^{\prime}$ nuclease allelic discrimination assays with allele-specific MGB probes (TaqMan, Applied Biosystems). All DNA 
samples were managed by the laboratory of the Division of Nephrology and Dialysis, San Raffaele Hospital (Milan, Italy).

\section{Expression analysis}

The study of human kidney samples was approved by the San Raffaele Scientific Institute Ethical Committee. Written informed consent was obtained from all patients. Human kidney cortex samples, derived from human nephrectomy because of tumors, were stored at the Pathology Department of San Raffaele Hospital. The kidney portions here used were verified to be histologically normal. The obtained samples were immediately immersed in RNAlater buffer (Ambion Inc.) at $4^{\circ} \mathrm{C}$ for 36 hours and then stored at $-80^{\circ} \mathrm{C}$. Genomic DNA was extracted with QIAamp DNA Mini Kit (Qiagen). All samples were genotyped for LSS rs2254524 SNP and then selected for the homozygous states. Definitively, total RNA was isolated from samples CC major and AA minor homozygous with mirVana miRNA Isolation Kit (Applied Biosystems). RNA concentration was obtained from $\mathrm{A}_{260}$ (absorbance at $260 \mathrm{~nm}$ ) measurements, and RNA integrity was checked on agarose gel. Sixteen samples displayed a good-quality RNA. To avoid the presence of genomic DNA, we treated all samples with DNase Deoxyribonuclease I, Amplification Grade (DNase I, Amp Grade, Invitrogen) before the reverse transcription reaction of $1 \mu \mathrm{g}$ of RNA with High-Capacity cDNA Reverse Transcription Kit (Applied Biosystems). mRNA expression of LSS gene was quantified by quantitative reverse transcription polymerase chain reaction (QRT-PCR) on the ABI Prism 7900 HT Sequence Detection System (Applied Biosystems) with the $2^{-\Delta \Delta C T}$ method. Primers and probes were obtained from Applied Biosystems Assay Gene Expression Product LSS. Because of its stable expression in kidney cortex samples, glyceraldehyde-3-phosphate dehydrogenase (GAPDH) reference gene was used for the normalization of quantification of mRNA expression. Real-time PCR was in a total volume of $20 \mu \mathrm{l}$ starting from complementary DNA (cDNA) template (20 ng of RNA converted to cDNA). No template control and calibrator were used in each run. All reactions were run in triplicates. Values are expressed as the amount of each cDNA normalized to GAPDH and to calibrator sample.

For the H295R cell culture transfection and analysis, an expression vector for LSS (642Val variant, obtained from ImaGenes $\mathrm{GmbH}$ ) was mutagenized with oligonucleotide 5 '-cgaggagcggcgttatttgcagagtgcc to generate the mutant variant 642Leu. H295R adrenocortical cells were transiently transfected with Nucleofector technology (Amaxa Biosystems). Three million cells were electroporated with $4 \mu \mathrm{g}$ of recombinant plasmids and collected after 48 hours for mRNA, protein, and activity quantification. For ouabain quantification in the medium, cells were serum-starved for 24 hours after transfection with 1:10 diluted medium and incubated for an additional 72 hours. Real-time quantitative PCR Target mRNA for LSS was quantified by real-time PCR detection with specific primers (forward: $5^{\prime}$-ttgcttcacctacggcacct and reverse: $5^{\prime}$ aggaagtcacaggcccgg) and MGB probe ( $5^{\prime}$-taccgagatgggactgc). Target mRNA levels were normalized to GAPDH and expressed as arbitrary units relative to mock levels. For SDS-polyacrylamide gel electrophoresis and immunoblotting, $5 \mu \mathrm{g}$ of total proteins was lysed with Laemmli sample buffer and analyzed by Western blotting with LSS primary antibody (OSC G-18, sc-83294, Santa Cruz Biotechnology Inc.), followed by 1-hour incubation with fluorescent secondary antibody (Alexa Fluor), and then analyzed and quantified by Odyssey Infrared Imaging Detection System (LI-COR Biosciences). Quantitative analysis of the fluorescent signal for LSS protein was reported as integrated intensity of fluorescent signal and expressed as arbitrary units relative to mock levels. LSS activity was measured in transfected cells after incubation with $1 \mu \mathrm{Ci}$ of $\left[{ }^{14} \mathrm{C}\right]$ acetate at $37^{\circ} \mathrm{C}$ for 3 hours. Cells were collected and lipids were saponified. After extraction and drying, lipids were separated on silica gel thin-layer chromatography plates, with hexane-ethyl ether-acetic acid (60:40:1) as described (40). The plates were autoradiographed to localize radioactive products. Spots of lanosterol and squalene monoepoxide were counted. Activity was expressed as nanomoles of acetate incorporated in lanosterol per hour per milligram of protein and corrected for squalene monoepoxide content. The supernatant from $12 \times 10^{6}$ transfected cells was collected for any individual ouabain quantification. Ouabain was determined by radioimmunoassay on $\mathrm{C}$-18-extracted samples with a specific antiserum, as described (41). Ouabain levels were expressed as picomoles per liter per microgram of cell proteins.

\section{Statistical analysis}

For statistical analysis of expression data in human kidneys, normalized gene expression data were derived from automatic analysis with RQ Manager 1.2 software (Applied Biosystems). ANOVA (analysis of variance) was used to compute $P$ value in univariate models for evaluation of the effect of SNP genotype on LSS expression. For H295R cell culture transfection, data were expressed as means \pm SEM. Statistical comparisons were analyzed by Student's $t$ test or one-way ANOVA. A $P$ value of 0.05 was chosen for statistical significance.

For genotyping, TagSNP selection in HSD3B1 gene was based on linkage disequilibrium $\left(r^{2}>0.8\right)$ in HapMap Rel22/Phase II relative to European population and was performed with Tagger (42) in Haploview 3.2 software package (43).

With general linear model (GLM) analysis, we tested whether the effect of the variable placebo/therapy on the dependent variable DSBP5_0 (decrease in SBP at the end of 5 weeks of treatment) depends on one or more SNPs in candidate genes. We reported the significance of the model and of the genes $\times$ therapy interaction. To show the trend of DSBP5_0 across the three genotypes of each SNP in placebo and rostafuroxin, we performed a one-way ANOVA in placebo and rostafuroxin separately, with DSBP5_0 as the dependent variable and genotypes as independent variables. ANOVA within treatment group also included trend analysis to look for a significant linear relationship between genotypes (additive model). The magnitude of therapy effect in a specific genotypic group (defined by the presence of a single genotype or by genotypic combination) was evaluated by ANOVA, with variation of SBP as the dependent variable and treatment as the independent one. $r^{2}$ represents the amount of variance explained by the treatment in the specific genotypic environment.

The FDR method (44) was applied to adjust for multiple comparisons. The FDR provides the expected ratio of erroneous rejections of the null hypothesis compared with the total number of rejected hypotheses. FDR values were calculated separately for 10 tests (that is, total number of SNPs studied) and for 18 tests (that is, total number of evaluated interactions), assessing the association between each genotypic group and the response to therapy or placebo. Tests were deemed significant at an FDR level of 0.10 .

The genetic profile shown in Table 3 has been obtained with a linear combination of the genotype interactions given in this table and in Fig. 2A. To evaluate the capacity of this profile to discriminate responders from nonresponders to rostafuroxin, we applied logistics regression on the genotype interactions reading the phenotype into a binomial trait. The magnitude of relationship is expressed in terms of odds ratio. Responders to the treatment are those patients with a decrease of DSBP5_0 
of $\leq-11.7 \mathrm{mmHg}$ (first tertile), and nonresponders are those patients with a DSBP5_0 variation of $>-11.7 \mathrm{mmHg}$.

Data are represented as box plots (Fig. 2), which contain the middle $50 \%$ of the data. The upper edge (hinge) of the box indicates the 75th percentile of the data set, and the lower hinge indicates the 25th percentile. The range of the middle two quartiles is the interquartile range. The line in the box indicates the median value. The end of the vertical lines or "whiskers" indicates the minimum and maximum data values.

\section{SUPPLEMENTARY MATERIAL}

www.sciencetranslationalmedicine.org/cgi/content/full/2/59/59ra87/DC1

Text

References

Excel File 1. Raw data from study 2003.

Excel File 2. Raw data from study 1 rostafuroxin.

Excel File 3. Raw data from study $2 \mathrm{HCTZ}$.

Excel File 4. Raw data from study 2 losartan.

\section{REFERENCES AND NOTES}

1. M. Ferrandi, I. Molinari, L. Torielli, G. Padoani, S. Salardi, M. P. Rastaldi, P. Ferrari, G. Bianchi, Adducin- and ouabain-related gene variants predict the antihypertensive activity of rostafuroxin. Part 1: Experimental studies. Sci. Transl. Med. 2, 59ra86 (2010).

2. G. Bianchi, Genetic variations of tubular sodium reabsorption leading to "primary" hypertension: From gene polymorphism to clinical symptoms. Am. J. Physiol. Regul. Integr. Comp. Physiol. 289, R1536-R1549 (2005).

3. L. Citterio, C. Lanzani, P. Manunta, G. Bianchi, Genetics of primary hypertension: The clinical impact of adducin polymorphisms. Biochim. Biophys. Acta 1802, 1285-1298 (2010).

4. A. C. Guyton, T. G. Coleman, H. J. Granger, Circulation: Overall regulation. Annu. Rev. Physiol. 34, 13-46 (1972).

5. S. Swart, R. F. Bing, J. D. Swales, H. Thurston, Plasma renin in long-term diuretic treatment of hypertension: Effect of discontinuation and restarting therapy. Clin. Sci. 63, 121-125 (1982).

6. J. R. Paull, R. E. Widdop, Persistent cardiovascular effects of chronic renin-angiotensin system inhibition following withdrawal in adult spontaneously hypertensive rats. J. Hypertens. 19, 1393-1402 (2001).

7. H. M. Qazzaz, Z. Cao, D. D. Bolanowski, B. J. Clark, R. Valdes Jr., De novo biosynthesis and radiolabeling of mammalian digitalis-like factors. Clin. Chem. 50, 612-620 (2004).

8. I. A. de Lannoy, M. Silverman, The MDR1 gene product, P-glycoprotein, mediates the transport of the cardiac glycoside, digoxin. Biochem. Biophys. Res. Commun. 189, 551-557 (1992).

9. W. Schoner, G. Scheiner-Bobis, Endogenous and exogenous cardiac glycosides: Their roles in hypertension, salt metabolism, and cell growth. Am. J. Physiol. Cell Physiol. 293, C509-C536 (2007).

10. J. R. Murrell, J. D. Randall, J. Rosoff, J. L. Zhao, R. V. Jensen, S. R. Gullans, G. T. Haupert Jr., Endogenous ouabain: Upregulation of steroidogenic genes in hypertensive hypothalamus but not adrenal. Circulation 112, 1301-1308 (2005).

11. J. A. Staessen, T. Kuznetsova, R. Acceto, A. Bacchieri, E. Brand, M. Burnier, H. Celis, L. Citterio, P. W. de Leeuw, J. Filipovský, A. Fournier, K. Kawecka-Jaszcz, P. Manunta, Y. Nikitin, E. T. O'Brien, J. Redón, L. Thijs, P. Ferrari, G. Valentini, G. Bianchi, OASIS-HT: Design of a pharmacogenomic dose-finding study. Pharmacogenomics 6, 755-775 (2005).

12. G. Tripodi, L. Citterio, T. Kouznetsova, C. Lanzani, M. Florio, R. Modica, E. Messaggio, J. M. Hamlyn, L. Zagato, G. Bianchi, J. A. Staessen, P. Manunta, Steroid biosynthesis and renal excretion in human essential hypertension: Association with blood pressure and endogenous ouabain Am. J. Hypertens. 22, 357-363 (2009).

13. K. L. Fung, M. M. Gottesman, A synonymous polymorphism in a common MDR1 (ABCB1) haplotype shapes protein function. Biochim. Biophys. Acta 1794, 860-871 (2009).

14. M. Cwynar, J. A. Staessen, M. Tichá, T. Nawrot, L. Citterio, T. Kuznetsova, W. Wojciechowska, K. Stolarz, J. Filipovský, K. Kawecka-Jaszcz, T. Grodzicki, H. A. Struijker-Boudier, L. Thijs, L. M. Van Bortel, G. Bianchi; European Project On Genes in Hypertension (EPOGH) Investigators, Epistatic interaction between $\alpha$ - and $\gamma$-adducin influences peripheral and central pulse pressures in white Europeans. J. Hypertens. 23, 961-969 (2005).

15. C. Lanzani, L. Citterio, M. Jankaricova, M. T. Sciarrone, C. Barlassina, S. Fattori, E. Messaggio, C. D. Serio, L. Zagato, D. Cusi, J. M. Hamlyn, A. Stella, G. Bianchi, P. Manunta, Role of the adducin family genes in human essential hypertension. J. Hypertens. 23, 543-549 (2005).
16. P. Manunta, M. Maillard, C. Tantardini, M. Simonini, C. Lanzani, L. Citterio, P. Stella, N. Casamassima, M. Burnier, J. M. Hamlyn, G. Bianchi, Relationships among endogenous ouabain, $\alpha$-adducin polymorphisms and renal sodium handling in primary hypertension. J. Hypertens. 26, 914-920 (2008).

17. J. A. Staessen, L. Thijs, K. Stolarz-Skrzypek, A. Bacchieri, J. Burton, E. Degli Espositi, P. W. de Leeuw, T. M. Dluzniewski, N. Glorioso, A. Januszewics, P. Manunta, V. Milyagin, Y. Nikitin, M. Souček, C. Lanzani, L. Citterio, M. Timio, A. Tykarski, P. Ferrari, G. Valentini, K. Kawecka-Jaszcz, G. Bianchi, Main results of the Ouabain and Adducin for Specific Intervention on Sodium in Hypertension Trial (OASIS-HT): A randomized placebo-controlled phase 2 dose-finding study of rostafuroxin. Trials, in press.

18. P. Ferrari, L. Torielli, M. Ferrandi, G. Padoani, L. Duzzi, M. Florio, F. Conti, P. Melloni, L. Vesci, N. Corsico, G. Bianchi, PST2238: A new antihypertensive compound that antagonizes the long-term pressor effect of ouabain. J. Pharmacol. Exp. Ther. 285, 83-94 (1998).

19. P. Ferrari, M. Ferrandi, G. Tripodi, L. Torielli, G. Padoani, E. Minotti, P. Melloni, G. Bianchi, PST 2238: A new antihypertensive compound that modulates Na,K-ATPase in genetic hypertension. J. Pharmacol. Exp. Ther. 288, 1074-1083 (1999).

20. M. Boddi, L. Poggesi, M. Coppo, N. Zarone, S. Sacchi, C. Tania, G. G. Neri Serneri, Human vascular renin-angiotensin system and its functional changes in relation to different sodium intakes. Hypertension 31, 836-842 (1998).

21. B. M. Psaty, C. Doggen, H. L. Vos, J. P. Vandenbroucke, F. R. Rosendaal, Association of the $\alpha$-adducin polymorphism with blood pressure and risk of myocardial infarction. J. Hum. Hypertens. 14, 95-97 (2000).

22. A. C. Morrison, M. S. Bray, A. R. Folsom, E. Boerwinkle, ADD1 $460 \mathrm{~W}$ allele associated with cardiovascular disease in hypertensive individuals. Hypertension 39, 1053-1057 (2002).

23. B. M. Psaty, N. L. Smith, S. R. Heckbert, H. L. Vos, R. N. Lemaitre, A. P. Reiner, D. S. Siscovick, J. Bis, T. Lumley, W. T. Longstreth Jr., F. R. Rosendaal, Diuretic therapy, the $\alpha$-adducin gene variant, and the risk of myocardial infarction or stroke in persons with treated hypertension. JAMA 287, 1680-1689 (2002).

24. X. F. Dou, H. Y. Zhang, X. H. Huang, X. N. Liu, Z. Y. Ju, K. Sun, D. W. Wang, Y. H. Liao, A. Q. Ma, Z. M. Zhu, B. R. Zhao, J. Z. Zhao, Y. Song, L. Zhang, R. T. Hui, $\alpha$-Adducin gene G/W460 polymorphism is associated with intracerebral hemorrhage in Chinese. Zhonghua Yi Xue Za Zhi 84, 186-188 (2004).

25. Y. Li, L. Thijs, T. Kuznetsova, L. Zagato, H. Struijker-Boudier, G. Bianchi, J. A. Staessen, Cardiovascular risk in relation to $\alpha$-adducin Gly460Trp polymorphism and systolic pressure: A prospective population study. Hypertension 46, 527-532 (2005).

26. M. J. van Rijn, M. J. Bos, M. Yazdanpanah, A. Isaacs, A. Arias-Vásquez, P. J. Koudstaal, A. Hofman, J. C. Witteman, C. M. van Duijn, M. M. Breteler, $\alpha$-Adducin polymorphism, atherosclerosis, and cardiovascular and cerebrovascular risk. Stroke 37, 2930-2934 (2006).

27. M. Yazdanpanah, F. A. Sayed-Tabatabaei, A. Hofman, Y. S. Aulchenko, B. A. Oostra, B. H. C. Stricker, H. A. Pols, S. W. Lamberts, J. C. Witteman, J. A. Janssen, C. M. van Duijn, The $\alpha$-adducin gene is associated with macrovascular complications and mortality in patients with type 2 diabetes. Diabetes 55, 2922-2927 (2006).

28. S. H. Cha, H. T. Kim, Y. Jang, S. Park, J. J. Kim, M. Y. Song, J. H. Park, H. J. Ryu, H. Y. Park, S. J. Yoon, K. Kimm, J. K. Lee, B. Oh, Association of $\alpha$-adducin Gly460Trp polymorphism with coronary artery disease in a Korean population. J. Hypertens. 25, 2413-2420 (2007).

29. M. H. Zafarmand, Y. T. van der Schouw, D. E. Grobbee, P. W. de Leeuw, M. L. Bots, $\alpha$-Adducin Gly460Trp variant increases the risk of stroke in hypertensive Dutch women. Hypertension $\mathbf{5 1}$ 1665-1670 (2008).

30. T. Gerhard, Y. Gong, A. L. Beitelshees, X. Mao, M. T. Lobmeyer, R. M. Cooper-DeHoff, T. Y. Langaee, N. J. Schork, M. D. Shriver, C. J. Pepine, J. A. Johnson; INVEST Investigators, $\alpha$-Adducin polymorphism associated with increased risk of adverse cardiovascular outcomes: Results from GENEtic Substudy of the INternational VErapamil SR-trandolapril STudy (INVESTGENES). Am. Heart J. 156, 397-404 (2008).

31. P. Manunta, M. Ferrandi, G. Bianchi, J. M. Hamlyn, Endogenous ouabain in cardiovascular function and disease. J. Hypertens. 27, 9-18 (2009).

32. A. J. Muslin, MAPK signalling in cardiovascular health and disease: Molecular mechanisms and therapeutic targets. Clin. Sci. 115, 203-218 (2008).

33. D. K. Arnett, S. A. Claas, A. I. Lynch, Has pharmacogenetics brought us closer to 'personalized medicine' for initial drug treatment of hypertension? Curr. Opin. Cardiol. 24, 333-339 (2009).

34. J. A. Johnson, Pharmacogenomics of antihypertensive drugs: Past, present and future. Pharmacogenomics 11, 487-491 (2010).

35. P. C. Phillips, Epistasis-the essential role of gene interactions in the structure and evolution of genetic systems. Nat. Rev. Genet. 9, 855-867 (2008).

36. D. B. Goldstein, Common genetic variation and human traits. N. Engl. J. Med. 360, 1696-1698 (2009).

37. D. S. Wald, M. Law, J. K. Morris, J. P. Bestwick, N. J. Wald, Combination therapy versus monotherapy in reducing blood pressure: Meta-analysis on 11,000 participants from 42 trials. Am. J. Med. 122, 290-300 (2009).

38. http://clinicaltrial.gov.

39. International HapMap Project; http://www.hapmap.org. 
40. A. Villagra, N. Ulloa, X. Zhang, Z. Yuan, E. Sotomayor, E. Seto, Histone deacetylase 3 downregulates cholesterol synthesis through repression of lanosterol synthase gene expression. J. Biol. Chem. 282, 35457-35470 (2007).

41. M. Ferrandi, P. Manunta, S. Balzan, J. M. Hamlyn, G. Bianchi, P. Ferrari, Ouabain-like factor quantification in mammalian tissues and plasma: Comparison of two independent assays. Hypertension 30, 886-896 (1997).

42. Tagger; http://www.broad.mit.edu/mpg/tagger.

43. Haploview 3.2; http://www.broad.mit.edu/mpg/haploview.

44. Y. Benjamini, Y. Hochberg, Controlling the false discovery rate: A practical and powerful approach to multiple testing. J. R. Stat. Soc. B 57, 289-300 (1995).

45. Acknowledgments: We thank R. Modica for cell culture experiments; N. Casamassima for help in DNA extraction; G. Dell'Antonio and R. Bertini for human kidney sample recruitment; A. Bacchieri and F. Cenci for calculation of carryover effect; P. Barassi for ouabain measurements; C. Scotti for technical assistance; and OASIS-HT Investigators: H. Celis (Leuven), J. Filipovský (Pilsen), M. Souček, J. Vítovec, J. Špinar (Brno), J. Barton, C. Kelly (Galway), G. Basso (Schio), E. Casiglia, V. Tikhonoff (Padua), E. Degli Espositi (Ravenna), M. Timio (Foligno), P. W. de Leeuw (Maastricht), M. Krekels (Sittard), M. Diuzniewski, A. Januszewicz (Warsaw), T. Grodzicki, K. Kawecka-Jaszcz, A. Olszanecka, K. Stolarz-Skrzpek, M. Klocek, W. Piwowarska (Krakow), K. Narkiewicz, B. Wyrzkowski (Gdansk), A. Tykarski (Poznan), Y. Nikitin (Novosibirsk), V. Milyagin (Smolensk), S. Nedogoda (Volgograd), J. Brugljan, R. Acceto (Ljubljana), C. Calvo-Gomez, A. Pose-Reino (Santiago de Compostela), and F. Hernández-Menárgez (Murcia). Funding: This work was supported by sigma-tau and by Rostaquo/sigma-tau SAR IDZ 01/2009. Author contributions: C.L., N.G., P.M., G.A., and G.V. followed the clinical studies; G.T. and M.F. performed experiments in cultured cells; L.C. and S.D.C. performed the genotyping and human expression analysis; E.M. coordinated the biological sample collection of study 1 ; C.L., L.C., E.S., and F.M. performed the statistical analysis; P.M., J.A.S., P.F., and G.B. were involved in project planning; J.A.S., D.C., P.F., and G.B. participated in manuscript preparation. Competing interests: G.T., G.V., and P.F. are employees of Prassis sigma-tau. G.B. is a paid consultant to Prassis sigma-tau. C.L., L.C., N.G., P.M., E.S., S.D.C., E.M., J.A.S., D.C., F.M., and G.A. declare that they have no competing financial interests. Accession numbers: Study 1 (OASIS-HT study) is registered as clinical trial NCT 00415038 . The raw data for study 1, study 2, and study 2003 are available in the Supplementary Material.

Submitted 29 July 2010

Accepted 5 November 2010

Published 24 November 2010

10.1126/scitranslmed.3001814

Citation: C. Lanzani, L. Citterio, N. Glorioso, P. Manunta, G. Tripodi, E. Salvi, S. D. Carpini, M. Ferrandi, E. Messaggio, J. A. Staessen, D. Cusi, F. Macciardi, G. Argiolas, G. Valentini, P. Ferrari, G. Bianchi, Adducin- and ouabain-related gene variants predict the antihypertensive activity of rostafuroxin. Part 2: Clinical studies. Sci. Transl. Med. 2, 59ra87 (2010). 